\title{
The First Internal Electromagnetic Motion Monitoring Implementation For Stereotactic Liver Radiotherapy in China: Procedures and Preliminary Results
}

\author{
Zhongde Mu ( $\nabla$ mouzhongde@jszlyy.com.cn ) \\ Jiangsu Cancer Hospital https://orcid.org/0000-0002-1984-4049 \\ Qi Wang \\ Jiangsu Cancer Hospital \\ Chang Guo \\ Jiangsu Cancer Hospital \\ Yong Feng \\ Jiangsu Cancer Hospital \\ Hongcheng Gu \\ Southeast University School of Biological Sciences and Medical Engineering \\ Zhenyu Zhai \\ Jiangsu Cancer Hospital \\ Jianfeng Wu \\ Jiangsu Cancer Hospital \\ Xia He \\ Jiangsu Cancer Hospital
}

\section{Research Article}

Keywords: Radiotherapy, Stereotactic Liver Radiotherapy, Internal Electromagnetic Motion Monitoring, Beacon Transponder, Motion Management

Posted Date: March 31st, 2021

DOI: https://doi.org/10.21203/rs.3.rs-330518/v1

License: (9) (1) This work is licensed under a Creative Commons Attribution 4.0 International License. Read Full License 
Version of Record: A version of this preprint was published at Journal of Cancer Research and Clinical Oncology on July 5th, 2021. See the published version at https://doi.org/10.1007/s00432-021-03726-z. 


\section{Abstract}

Background. Respiratory motion may compromise the dose delivery accuracy in liver stereotactic body radiation therapy (SBRT). Motion management can improve treatment delivery. However, external surrogate signal may be unstable and inaccurate. This study reports the first case of liver SBRT based on internal electromagnetic motion monitoring in China.

Materials and Methods. The patient with a primary liver cancer was treated with respiratory-gated SBRT guided by three implanted electromagnetic transponders. The treatment was carried out in breath-hold end-exhale with beam-on when the centroid of the three transponders drifted within $5 \mathrm{~mm}$ (left-right (LR), anterior-posterior (AP) and cranio-caudal (CC) directions) from the planned position. The motion monitoring treatments were delivered in breath-hold end-exhale mode with the energy of $6 \mathrm{MV}$ in FFF mode with 1200 monitor units (MU) per minute. For each fraction, QA results, intertransponder distances, geometric checks as well as tumor motion logs were explicitly recorded.

Results. Comparing with the plan data, distance variances between each two transponders were $-0.056 \pm 0.032 \mathrm{~cm}, 0.017 \pm 0.033 \mathrm{~cm}$ and $-0.082 \pm 0.068 \mathrm{~cm}$. Geometric residual, the pitch, roll and yaw angles were $0.048 \pm 0.021 \mathrm{~cm}$ (threshold $0.2 \mathrm{~cm}$ ), $2.17^{\circ} \pm 1.85^{\circ}$ (threshold $10^{\circ}$ ), $-2.42^{\circ} \pm 1.51^{\circ}$ (threshold $10^{\circ}$ ) and $1.67^{\circ} \pm 1.07^{\circ}$ (threshold $10^{\circ}$ ), respectively. The delivery time of the five fields were $13.8 \mathrm{~s}, 13.1 \mathrm{~s}, 11.18$ $\mathrm{s}, 11.57 \mathrm{~s}, 11.62 \mathrm{~s}$ with the average value of $12.254 \pm 1.13 \mathrm{~s}$. Treatment duration of each fraction ranged from 6.22 minutes to 21.43 minutes, with the average value of $11.25 \pm 5.03$ minutes.

Conclusions. The first case of liver SBRT patient of China based on internal electromagnetic motion monitoring was performed. The system had a high tracking accuracy, and it did not delay the treatment time. In addition, the patient did not show any severe side effects except for $I^{\circ}$ myelotoxicity. The internal electromagnetic motion monitoring system provides a real-time and direct way to track liver tumor targets.

\section{Introduction}

Due to large and irregularity respiratory movements, motion management is still a challenge for stereotactic liver radiotherapy. It is reported that liver can move up to $3.5 \mathrm{~cm}$ and even $7.5 \mathrm{~cm}$ due to normal and deep inspiration ${ }^{(1-4)}$. Besides, liver motion also includes its deformation, patients' inconsistent motion pattern between treatment fractions, making it more challenging for liver radiotherapy treatments.

Nowadays, cone beam computed tomography (CBCT) and magnetic resonance imaging (MRI) guided treatment technologies have been deployed on linear accelerators (linacs) for image guided radiation therapy ${ }^{(5-7)}$. However, liver targets are not typically distinct based on $x$-ray technologies, furthermore, CBCT could result in excess radiation dose to the patient ${ }^{(8,9)}$. In consideration of avoiding excessive dose, СBCT are often applied for localization rather than continuous tracking. Although MRI guided treatment 
technologies can provide continuous tracking without excessive imaging dose, such systems are very expensive and thus hinder their extensive application ${ }^{(10)}$.

In recent years, many motion management technologies have been developed for tumor radiotherapy, such as Active Breathing Coordinator ${ }^{\text {TM }}$ (ABC, ELEKTA, Sweden) ${ }^{(11)}$, Real-time Position Management ${ }^{\text {TM }}$ (RPM, Varian Medical Systems, USA) ${ }^{(12)}$,Optical Surface Management System (OSMS, Varian Medical Systems, USA) ${ }^{(13)}$, etc. All these methods are non-invasive (external) approaches to track inspiratory change or in vitro optical markers as surrogates for the tumor position. External motion monitoring is readily accessible and can automatically halt (gating) the treatment if external markers movements exceed the preplanned thresholds. However, the correlation between external and internal motion is unstable, and sometimes the external monitoring can be inaccurate ${ }^{(14,15)}$. Therefore, it is optimal that gating directly relies on internal motion monitoring, and the necessity for direct internal movement monitoring has become essential.

Recently, an internal electromagnetic motion monitoring system has been developed, which is a real-time and direct approach to localize and track targets in prostate cancer ${ }^{(16,17)}$, lung cancer ${ }^{(18,19)}$ and pancreatic cancer ${ }^{(20)}$, etc. This system received Conformité Européenne marking and US Food and Drug Administration clearance for general implantation in soft issues in 2014. Thereafter, the first case of liver motion management radiotherapy based on this system was deployed at University of Louisville in March $2015^{(21,22)}$, and the first clinical experience including accuracy of dosimetry and geometry were reported from Aarhus University Hospital in April 2015(23). High dosimetric accuracy and benefits for liver stereotactic body radiation therapy (SBRT) based on this system were successively reported in the last decade ${ }^{(24-26)}$.

It seemed that, because of low popularity rate of this system, invasive puncture and ignorant selection of appropriate patients, there was no liver SBRT case reported in China since this system appeared. In consideration of challenges in liver radiotherapy and advantages of this tracking system, we attempted to implement this system for motion management of liver treatments. To our knowledge we are the first institution to use this system for liver radiotherapy in China. In this paper on our first experiences, a summary medical physics report for the first one liver radiotherapy based on this system was given. Operation procedures and a home-made motion tracking video signal synchronizing device were also elaborately described.

\section{Material And Methods}

Written informed consent was obtained from the patient for the publication of any potentially identifiable images or data included in this article. The patient was a 44-year-old male and diagnosed with liver cancer in 2012. Since then, he received 20 times of interventional therapy and 4 times of microwave treatment. In addition, he has also taken adefovir dipivoxil and entecavir for anti-hepatitis B virus therapy since 2017. The patient is healthy before, and has no history of heart disease or hypertension, no allergy 
history of food and drugs, no operative history and no disease history in other system. In March 2019, a routine medical examination report showed that he had $3.3 \mathrm{ng} / \mathrm{ml}$ AFP and $2.74 \mathrm{~nm} / \mathrm{ml} \mathrm{CEA}$, respectively. An epigastric CT examination indicated that a possibility of lesions in lower left inner lobe of liver. Then, the patient considered receiving a radiotherapy treatment for a primary liver cancer (CTV: $25 \mathrm{~cm}^{3}$ ). Guided by CT simulator, three electromagnetic transponders were implanted percutaneously with $17 \mathrm{G}$ needles in close proximity to the target. A breath-hold end-exhale scan and a free-breath scan, both with $1 \mathrm{~mm}$ slice thickness, were acquired five days after transponder implantation. An in-house developed vacuum cushion was used for patient immobilization and no abdominal compression was applied.

The planning target volume (PTV) was formed by expanding the CTV by $5 \mathrm{~mm}$ margins in the left-right (LR), anterior-posterior (AP) and cranio-caudal (CC) directions. The plan was a five-field intensity modulated radiation therapy (IMRT) with a mean dose of $100 \%$ of the prescription dose cover $95 \%$ volumes of PTV. Fraction dose of $4 \mathrm{~Gy}$ with 12 fractions were prescribed to the $100 \%$ dose level. All three transponders were marked in the plan, and their center-of-mass position was recorded as the isocenter.

The motion monitoring treatments were delivered in breath-hold end-exhale mode with a VitalBeam Linac equipped with the tracking system. The energy was $6 \mathrm{MV}$ in FFF mode with a dose rate of 1200 monitor units (MU) per minute. Prior to every fraction treatment, a QA test was made to keep the accuracy of the tracking system isocenter.

A home-made tracking video signal synchronizing device was equipped to transmit the video signals of the internal electromagnetic motion, OSMS (Optical Surface Monitoring System) and RPM (Real-time Position Management) from the control room to the treatment room via a three-channel switch. The patients could observe his/her own motion signal and stabilize his/her respiratory movements simultaneously to adjust motion deviations towards zero.

\section{Results And Discussion}

Firstly, a throughout literature retrieve was carried out with respect to the keyword "calypso \& radiotherapy" via the Web of Science. It was found that only three articles were reported by Chinese institutions. Two of them were talking about the accuracy evaluation ${ }^{(27,28)}$, and the third was focusing on algorithms comparison for localizing prostate tumor ${ }^{(29)}$. All of them were medical physical researches rather than clinical experiences. Therefore, based on the aforementioned literature search, it can be concluded that we are the first institution to utilize internal tracing system for precise liver radiotherapy in China. The first implement of internal motion monitoring for liver treatment in the world was carried out in March 2015, and the first paper was published in April 2015 by two different groups.

FigureS1 (in Supplementary Information) is the flowchart of implementing the internal tracing system for liver radiotherapy. It can be seen that the main processes are the same as the regular radiotherapy except for beacon implantation, beacon marking, etc. The main differences to regular radiotherapy are that beacons for tracking must be implantation before CT positioning, and then, the beacons are tracked 
during the treatment. Apart from that, QA and array setup must be done prior to tracking and treatment to make sure the setup offsets distributing in an acceptable range. The QA fixture is a white cube block containing 3 beacon transponders for performing the QA procedure, and the array is a plate planted above the fixture or the patient for tracking the beacons movements inside the patient's body.

Figure 1 shows the comparison of CT images of the same patient by a free-breath scan and a breath-hold end-exhale scan. It can be seen that the liver has a movement of up to $2 \mathrm{~cm}$ which is a big challenge for non-motion managed radiotherapy. Although respiration management techniques such as ABC (Active Breathing Coordinator) and RPM (real-time position management) were utilized for assisting precise radiotherapy, these were all performed indirectly. The internal electromagnetic motion management is working in a direct way by monitoring the real-time signals transmitted by the beacons which are elliptic cylinders with an $8.7 \mathrm{~mm}$ length and a $1.3 \mathrm{~mm}$ diameter, and were implanted into the patient's body by $17 \mathrm{G}$ needles as shown in Fig. $1 \mathrm{C}$ and D.

CT positioning, cancer and organs at risk contouring were the same as regular processes. The slice thickness of CT images should be in the range of $1.0 \mathrm{~mm}$ to $1.5 \mathrm{~mm}$ to ensure that the beacons would not be lost in adjacent image slices. Prior to planning, beacons were found and marked by the "Calypso Beacon Detection" function in the Contouring Module (Eclipse 13.6, Varian Medical Systems, Inc. USA).

Figure 2 shows the planning and DVH results. It can be seen that the $100 \%$ isodose line has a good conformity with regard to the PTV. This can be attributed to that the PTV approximate spherical shape. Table S1 (in Supplementary Information) is a list of the dose statistical results for CTV, PTV, Liver and Spinal Cord. It can be noticed that mean doses for CTV and PTV have reached the prescription. Mean dose for liver and max dose for spinal cord were in a safe range. Besides, it can also be seen that max dose for CTV is lower than that of PTV, indicating that the max dose point located in PTV rather than CTV. As the dose was delivered in a breath-hold end-exhale phase, it can be assumed that it is still safe for the patient.

Before the plan delivery, several procedures should be done in advance. To ensure proper operation of the system, a QA procedure must be performed daily. The QA procedures in this article were done by the QA fixture, as shown in Fig. 3A. Subsequently, distance offsets of the measured position of the QA fixture isocenter from the calibrated isocenter position in the lateral, longitudinal and vertical directions were recorded. Finally, the isocenter offsets were calculated by the following equation:

$$
\text { Offset }=\sqrt{x^{2}+y^{2}+z^{2}}
$$

Where in the equation, $x, y$ and $z$ mean the offsets in the lateral, longitudinal and vertical directions, respectively. The isocenter offsets must be less than or equal to $0.2 \mathrm{~cm}$ to ensure the QA procedure to pass. By summarizing the 12 times of QA test results, it was shown that the tracking system has $0.108 \pm$ $0.026 \mathrm{~cm},-0.054 \pm 0.037 \mathrm{~cm}$ and $0.08 \pm 0.027 \mathrm{~cm}$ offsets in the lateral, longitudinal and vertical directions, respectively, as shown in Table S2 (in Supplementary Information). The isocenter offset, which 
was determined by the above three offsets, was $0.152 \pm 0.024 \mathrm{~cm}$. The QA results indicated that the tracking system was considerably stable during the 12 fractions, which ensured the isocenter stability for the subsequent patient and array setup.

After the patient and array setup were done, the system was detecting the intertransponder distances inside the patient's body prior to dose delivery. Table S3 (in Supplementary Information) shows the intertransponder distances offsets of the measured positions from the distances in the plan. Comparing with the plan data, distance variances between each two transponders were $-0.056 \pm 0.032 \mathrm{~cm}, 0.017 \pm$ $0.033 \mathrm{~cm}$ and $-0.082 \pm 0.068 \mathrm{~cm}$, indicating that the transponders variances were very small in the liver and guaranteed the accuracy of target tracking.

Positions of the three transponders were in further determining the geometric residual of the target, the pitch, roll and yaw angles, which were shown in Table S4 (in Supplementary Information), with the variances of $0.048 \pm 0.021 \mathrm{~cm}$ (threshold $0.2 \mathrm{~cm}$ ), $2.17^{\circ} \pm 1.85^{\circ}$ (threshold $10^{\circ}$ ), $-2.42^{\circ} \pm 1.51^{\circ}$ (threshold $10^{\circ}$ ) and $1.67^{\circ} \pm 1.07^{\circ}$ (threshold $10^{\circ}$ ), respectively. It was indicating that the target was located in the correct positions which was beneficial to the following treatments.

During the tracing procedure, the actual dose delivery time is very short, as shown in the light gray areas of Fig. 4A. The reason is that only the end-exhale phases were used for beam on. Although there was a gating interlock to control the beam on and off, the appropriate range in a respiratory cycle is so short, therefore, the delivery efficiency was reduced. In addition, therapists could see the liver tumor motion in real-time. When the patient was exhaling, curves of three directions were moving towards 0 . Meanwhile, therapists told the patient to hold the breath using the talkback system. Therefore, by using the endexhale phases and observing the tracking curves, doses could be precisely delivered.

Although breath-hold end-exhale could favor high dose delivery and reduce the overall treatment time, it is still a challenge for elder patients. Figure $4 \mathrm{~B}$ shows the third patient being treated in our hospital by the tracking system on 15th April, 2020, who was male with the age of 83 years old. It is clearly seen that it is very hard for the patient to hold-breath during the beam on cycle, and a big base-line drift could be seen in Fig. 4C. In sake of a more stable motion during breath-hold period, a tracking video signal synchronizing device was designed to transmit the video signals of the internal electromagnetic motion, OSMS and RPM from the control room to the treatment room via a three-channel switch. As is shown in Fig. 4B, the patient could observe his own target motion curves by a mini screen which is connected to a VGA signal cable, and the screen displayed exactly the same motion curves as that of the screens in the control room. Thus, the patient could make the tracking signal more stable during the beam-on phase by adjusting his own breath motion. By using the video signal synchronizing device, it is clearly seen from Fig. 4D that the motion curves are more stable than that in Fig. 4C. In short, the video signal synchronizing device could give to more stable breath-hold phases, accurate treatment positions of the target and less treatment durations for each fraction.

For the sake of investigating how much the tracking system could affect the treatment time, delivery time for each field and treatment duration for each fraction were recorded. The delivery time of the five fields 
were $13.8 \mathrm{~s}, 13.1 \mathrm{~s}, 11.18 \mathrm{~s}, 11.57 \mathrm{~s}, 11.62 \mathrm{~s}$ with the average value of $12.25 \pm 1.13 \mathrm{~s}$ (Fig. S2A, Supplementary Information). Therefore, the patient was keeping breath-hold for just five short time and was easily adapt to the treatment style in each fraction. It is also noted that base lines in the short delivery time almost have no drifts (Fig. 4A). Treatment durations of each fraction were also recorded from the beginning to the end of the tracking (Fig. S2B, Supplementary Information). The durations were ranging from 6.22 minutes to 21.43 minutes, with the average value of $11.25 \pm 5.03$ minutes. Due to CBCT scan, image registration and couch shifts, durations of the last two fraction were reach up to about 20 minutes. Even though, the tracking system did not notably lengthen the treatment time compared to the one without the system in our hospital. After the radiotherapy completed, the patient only showed $I^{\circ}$ myelotoxicity and became better with symptomatic treatment before discharging from the hospital.

\section{Conclusions}

To our knowledge we are the first institution to implement the internal electromagnetic motion monitoring system for motion management of liver radiotherapy in China. The system was stable during the treatments and had a high tracking accuracy, meanwhile, it did not delay the treatment time. A video signal synchronizing device was designed which could give rise to more stable breath-hold phases, accurate treatment positions of the target and less treatment durations for each fraction. The internal electromagnetic motion monitoring system is a new gating method for liver treatment, and provides a real-time and direct way to track targets compared to other motion management techniques such as OSMS, RPM and ABC. In addition, introduction of the system to linacs did not bring about severe side effects to patients. It is hoped that our first experience and preliminary results can provide helpful reference to liver SBRT colleagues in not only China but also all around the world.

\section{Declarations}

\section{Acknowledgments}

The authors thank the therapist group of the Radiotherapy Department of Jiangsu Cancer Hospital for their helpful advices and selfless assistance. The authors thank the School of Biomedical Science and Medical Engineering for their help in designing the video signal synchronizing device. The authors also thank Miss Vicky Chen for her careful proofreading.

\section{Compliance with Ethical Standards:}

Funding: This study was funded by China Postdoctoral Science Foundation (2018M632263), Southeast University - Nanjing Medical University Cooperative Research Project (2242018K3DN22) and Postdoctoral Foundation of Jiangsu Institute of Cancer Research (SZL201715).

Conflict of Interest: The authors declare that the research was conducted in the absence of any commercial or financial relationships that could be construed as a potential conflict of interest. 
Ethical approval: All procedures performed in studies involving human participants were in accordance with the ethical standards of the institutional and/or national research committee and with the 1964 Helsinki declaration and its later amendments or comparable ethical standards.

Informed consent: Informed consent was obtained from all individual participants included in the study.

\section{References}

1. Fernandes AT, Apisarnthanarax S, Yin L, Zou W, Rosen M, Plastaras JP, et al. Comparative assessment of liver tumor motion using cine-magnetic resonance imaging versus 4-dimensional computed tomography. Int J Radiat Oncol Biol Phys (2015) 91(5):1034-40. Epub 2015/04/04. doi: 10.1016/j.jijrobp.2014.12.048. PubMed PMID: 25832694.

2. Kirilova A, Lockwood G, Choi P, Bana N, Haider MA, Brock KK, et al. Three-dimensional motion of liver tumors using cine-magnetic resonance imaging. Int J Radiat Oncol Biol Phys (2008) 71(4):1189-95. Epub 2008/02/09. doi: 10.1016/j.jirobp.2007.11.026. PubMed PMID: 18258378.

3. Shimohigashi $\mathrm{Y}$, Toya R, Saito T, Ikeda O, Maruyama M, Yonemura $\mathrm{K}$, et al. Tumor motion changes in stereotactic body radiotherapy for liver tumors: an evaluation based on four-dimensional cone-beam computed tomography and fiducial markers. Radiat Oncol (2017) 12(1):61. Epub 2017/03/25. doi: 10.1186/s13014-017-0799-7. PubMed PMID: 28335794; PubMed Central PMCID: PMCPMC5364670.

4. Colvill E, Krieger M, Bosshard P, Steinacher P, Rohrer Schnidrig BA, Parkel T, et al. Anthropomorphic phantom for deformable lung and liver CT and MR imaging for radiotherapy. Phys Med Biol (2020) 65(7):07NT2. Epub 2020/02/12. doi: 10.1088/1361-6560/ab7508. PubMed PMID: 32045898.

5. Sweeney RA, Seubert B, Stark S, Homann V, Müller G, Flentje M, et al. Accuracy and inter-observer variability of $3 \mathrm{D}$ versus $4 \mathrm{D}$ cone-beam $\mathrm{CT}$ based image-guidance in SBRT for lung tumors. Radiation Oncology (2012) 7(1):81. doi: 10.1186/1748-717X-7-81.

6. Lagendijk JJ, Raaymakers BW, Van den Berg CA, Moerland MA, Philippens ME, van Vulpen M. MR guidance in radiotherapy. Phys Med Biol (2014) 59(21):R349-69. Epub 2014/10/17. doi: 10.1088/0031-9155/59/21/R349. PubMed PMID: 25322150.

7. Velec M, Tadic T, Xie J, Moseley JL, Patel T, Milosevic M, et al. Deformable Dose Accumulation for Hybrid CBCT-MRI Guided Adaptive Radiotherapy for Cervix Cancer. International Journal of Radiation

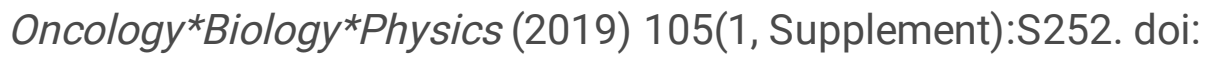
https://doi.org/10.1016/j.jijobp.2019.06.376.

8. Li Y, Netherton T, Nitsch PL, Balter PA, Gao S, Klopp AH, et al. Normal tissue doses from MV imageguided radiation therapy (IGRT) using orthogonal MV and MV-CBCT. J App/ Clin Med Phys (2018) 19(3):52-7. Epub 2018/03/04. doi: 10.1002/acm2.12276. PubMed PMID: 29500856; PubMed Central PMCID: PMCPMC5978715.

9. Quinn A, Holloway L, Cutajar D, Hardcastle N, Rosenfeld A, Metcalfe P. Megavoltage cone beam CT near surface dose measurements: potential implications for breast radiotherapy. Med Phys (2011) 38(11):6222-7. Epub 2011/11/04. doi: 10.1118/1.3641867. PubMed PMID: 22047387. 
10. Herrmann H, Seppenwoolde Y, Georg D, Widder J. Image guidance: past and future of radiotherapy. Radiologe (2019) 59(Suppl 1):21-7. Epub 2019/07/28. doi: 10.1007/s00117-019-0573-y. PubMed PMID: 31346650; PubMed Central PMCID: PMCPMC6914710.

11. Brock J, McNair HA, Panakis N, Symonds-Tayler R, Evans PM, Brada M. The use of the Active Breathing Coordinator throughout radical non-small-cell lung cancer (NSCLC) radiotherapy. Int J Radiat Oncol Biol Phys (2011) 81(2):369-75. Epub 2010/08/31. doi: 10.1016/j.ijrobp.2010.05.038. PubMed PMID: 20800379.

12. Lee TK, Ewald A, Schultz T, Park SY. SU-E-J-253: Evaluation of 4DCT Images with Correlation of RPM Signals to Tumor Motion for Respiratory-Gated Radiotherapy. Medical Physics (2014) 41(6Part10):216-. doi: 10.1118/1.4888307.

13. Ma Z, Zhang W, Su Y, Liu P, Pan Y, Zhang G, et al. Optical Surface Management System for Patient Positioning in Interfractional Breast Cancer Radiotherapy. Biomed Res Int (2018) 2018:6415497. Epub 2018/03/08. doi: 10.1155/2018/6415497. PubMed PMID: 29511688; PubMed Central PMCID: PMCPMC5817315.

14. Ge J, Santanam L, Yang D, Parikh PJ. Accuracy and consistency of respiratory gating in abdominal cancer patients. Int J Radiat Oncol Biol Phys (2013) 85(3):854-61. Epub 2012/06/22. doi: 10.1016/j.jjobp.2012.05.006. PubMed PMID: 22717241.

15. George R, Chung TD, Vedam SS, Ramakrishnan V, Mohan R, Weiss E, et al. Audio-visual biofeedback for respiratory-gated radiotherapy: impact of audio instruction and audio-visual biofeedback on respiratory-gated radiotherapy. Int J Radiat Oncol Biol Phys (2006) 65(3):924-33. Epub 2006/06/06. doi: 10.1016/j.jirobp.2006.02.035. PubMed PMID: 16751075.

16. Li J, Pollack A, Horwitz E, Buyyounouski M, Price R, Ma C. Clinical Experience on Localization and Real-Time Tracking of the Prostate During External Radiotherapy using Calypso ${ }^{\circledR}$ 4D Localization System. International Journal of Radiation Oncology*Biology*Physics (2007) 69(3). doi: 10.1016/j.jijrobp.2007.07.342.

17. Kupelian P, Willoughby T, Mahadevan A, Djemil T, Weinstein G, Jani S, et al. Multi-institutional clinical experience with the Calypso System in localization and continuous, real-time monitoring of the prostate gland during external radiotherapy. Int J Radiat Oncol Biol Phys (2007) 67(4):1088-98. Epub 2006/12/26. doi: 10.1016/j.jrobp.2006.10.026. PubMed PMID: 17187940.

18. Sawant A, Smith RL, Venkat RB, Santanam L, Cho B, Poulsen P, et al. Toward submillimeter accuracy in the management of intrafraction motion: the integration of real-time internal position monitoring and multileaf collimator target tracking. Int J Radiat Oncol Biol Phys (2009) 74(2):575-82. Epub 2009/03/31. doi: 10.1016/j.jijrobp.2008.12.057. PubMed PMID: 19327907.

19. Montanaro T, Nguyen DT, Keall PJ, Booth J, Caillet V, Eade T, et al. A comparison of gantry-mounted xray-based real-time target tracking methods. Med Phys (2018) 45(3):1222-32. Epub 2018/01/25. doi: 10.1002/mp.12765. PubMed PMID: 29363760.

20. Shinohara ET, Kassaee A, Mitra N, Vapiwala N, Plastaras JP, Drebin J, et al. Feasibility of electromagnetic transponder use to monitor inter- and intrafractional motion in locally advanced 
pancreatic cancer patients. Int J Radiat Oncol Biol Phys (2012) 83(2):566-73. Epub 2011/11/22. doi: 10.1016/j.jijrobp.2011.07.025. PubMed PMID: 22099029.

21. James J, Cetnar A, Nguyen V, Wang B. MO-F-CAMPUS-J-02: Commissioning of Radiofrequency Tracking for Gated SBRT of the Liver Using Novel Motion System. Medical Physics (2015) 42(6Part30):3582-. doi: 10.1118/1.4925482.

22. James J, Cetnar A, Dunlap NE, Huffaker C, Nguyen VN, Potts M, et al. Technical Note: Validation and implementation of a wireless transponder tracking system for gated stereotactic ablative radiotherapy of the liver. Med Phys (2016) 43(6):2794-801. Epub 2016/06/10. doi:

10.1118/1.4948669. PubMed PMID: 27277027.

23. Poulsen PR, Worm ES, Hansen R, Larsen LP, Grau C, Hoyer M. Respiratory gating based on internal electromagnetic motion monitoring during stereotactic liver radiation therapy: First results. Acta Oncol (2015) 54(9):1445-52. Epub 2015/07/23. doi: 10.3109/0284186X.2015.1062134. PubMed PMID: 26198651.

24. Bertholet J, Toftegaard J, Hansen R, Worm ES, Wan H, Parikh PJ, et al. Automatic online and real-time tumour motion monitoring during stereotactic liver treatments on a conventional linac by combined optical and sparse monoscopic imaging with kilovoltage x-rays (COSMIK). Phys Med Biol (2018) 63(5):055012. Epub 2018/03/09. doi: 10.1088/1361-6560/aaae8b. PubMed PMID: 29516868.

25. Worm ES, Hoyer M, Hansen R, Larsen LP, Weber B, Grau C, et al. A Prospective Cohort Study of Gated Stereotactic Liver Radiation Therapy Using Continuous Internal Electromagnetic Motion Monitoring. Int J Radiat Oncol Biol Phys (2018) 101(2):366-75. Epub 2018/03/22. doi:

10.1016/j.jijrobp.2018.02.010. PubMed PMID: 29559289.

26. Skouboe S, Poulsen PR, Muurholm CG, Worm E, Hansen R, Hoyer M, et al. Simulated real-time dose reconstruction for moving tumors in stereotactic liver radiotherapy. Med Phys (2019) 46(11):4738-48. Epub 2019/08/31. doi: 10.1002/mp.13792. PubMed PMID: 31468543.

27. Dai ZH, Zhang BL, Zhu L, Zhu YH, Yang G, Cai CY, et al. Evaluation of the accuracy of Calypso 4D electromagnetic localization system in Edge accelerator. Chinese Journal of Medical Physics (2018) 35(5):497-502. doi: 10.3969/j.issn.1005-202X.2018.05.001.

28. Dai Z, Zhang H, Xie Y, Zhu L, Zhang B, Cai C, et al. Validation of Geometric and Dosimetric Accuracy of Edge Accelerator Gating With Electromagnetic Tracking: A Phantom Study. IEEE Access (2019) 7:127693-702. doi: 10.1109/access.2019.2934858.

29. Zheng C, Dai JR. Comparison of Algorithms for Localizing Prostate Tumor Via Monoscopic X-ray Imaging. Chinese Journal of Medical Physics (2013) 30(6):4491-6. doi: 10.3969/j.issn.1005202X.2013.06.006.

\section{Figures}




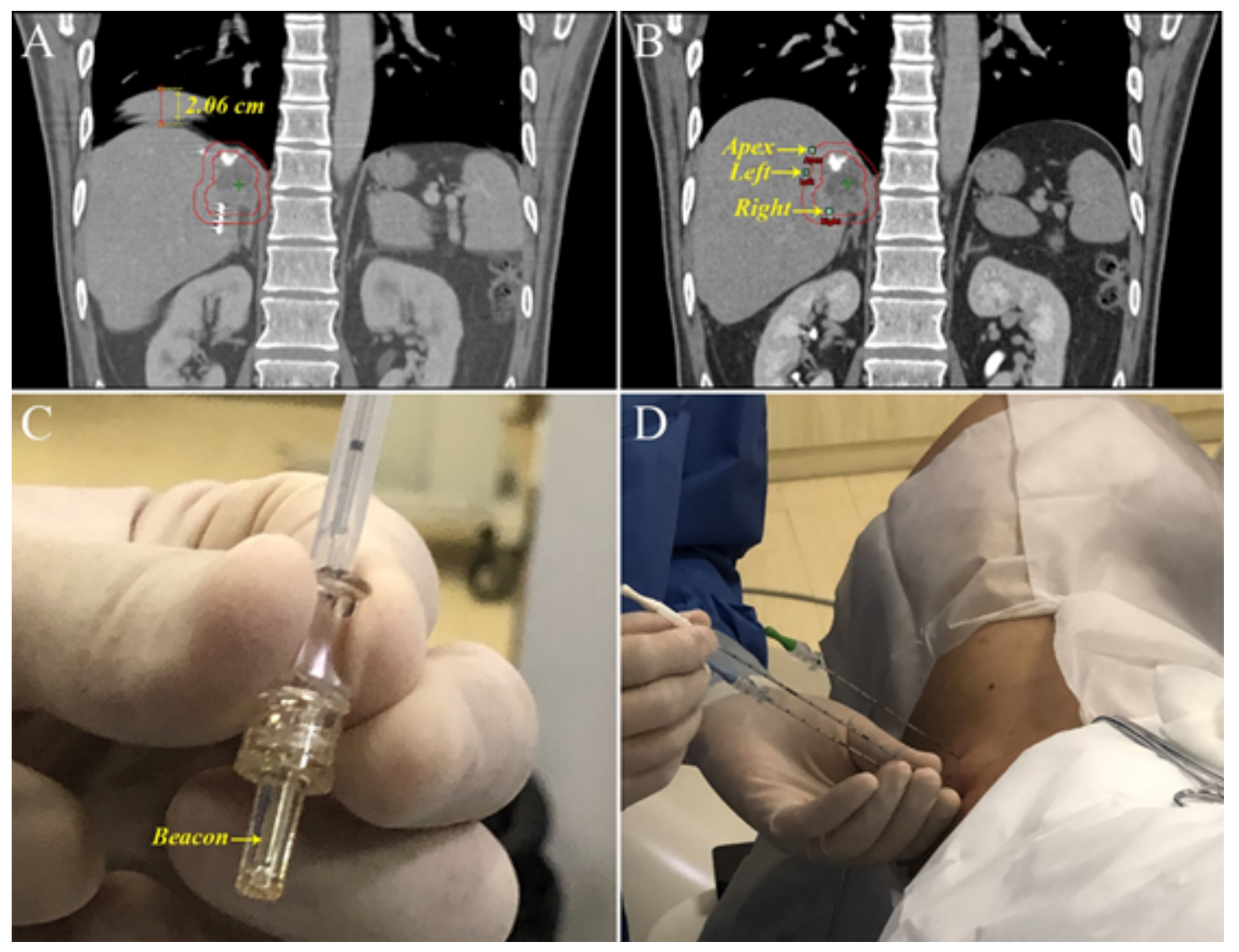

Figure 1

Coronal CT views of the patient by A) a free-breath scan and B) a breath-hold end-exhale scan. Note that the liver could move up to $2 \mathrm{~cm}$ due to respiration. C) Beacon in a $17 \mathrm{G}$ needle and $\mathrm{D}$ ) beacon implantation assisted by CT scans.

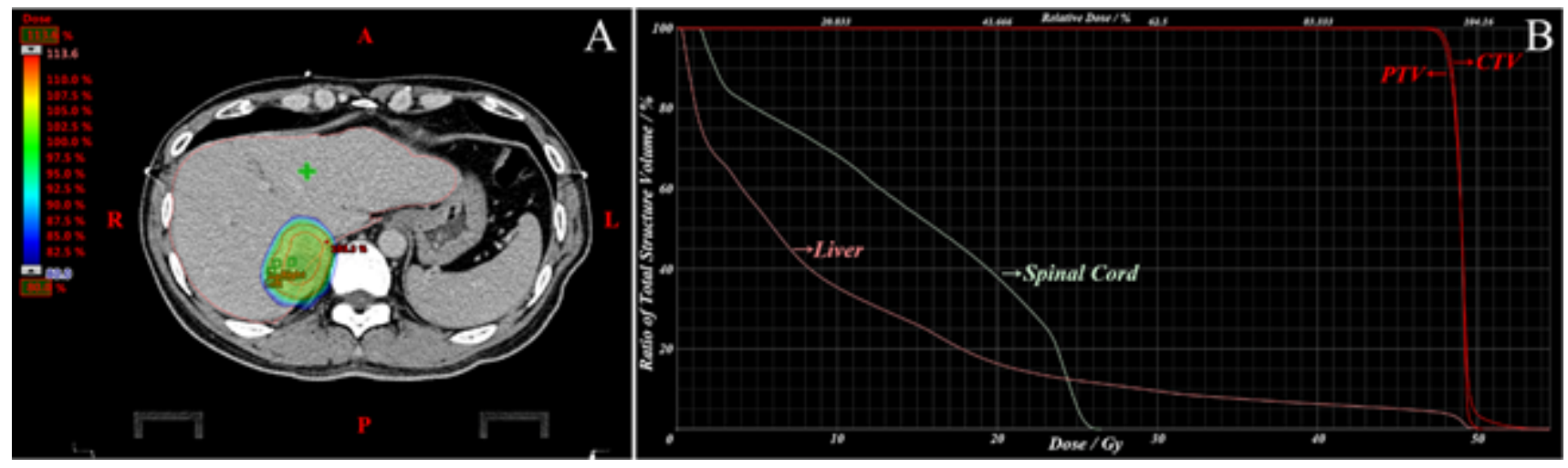

Figure 2

Planning (A) and DVH results (B). 


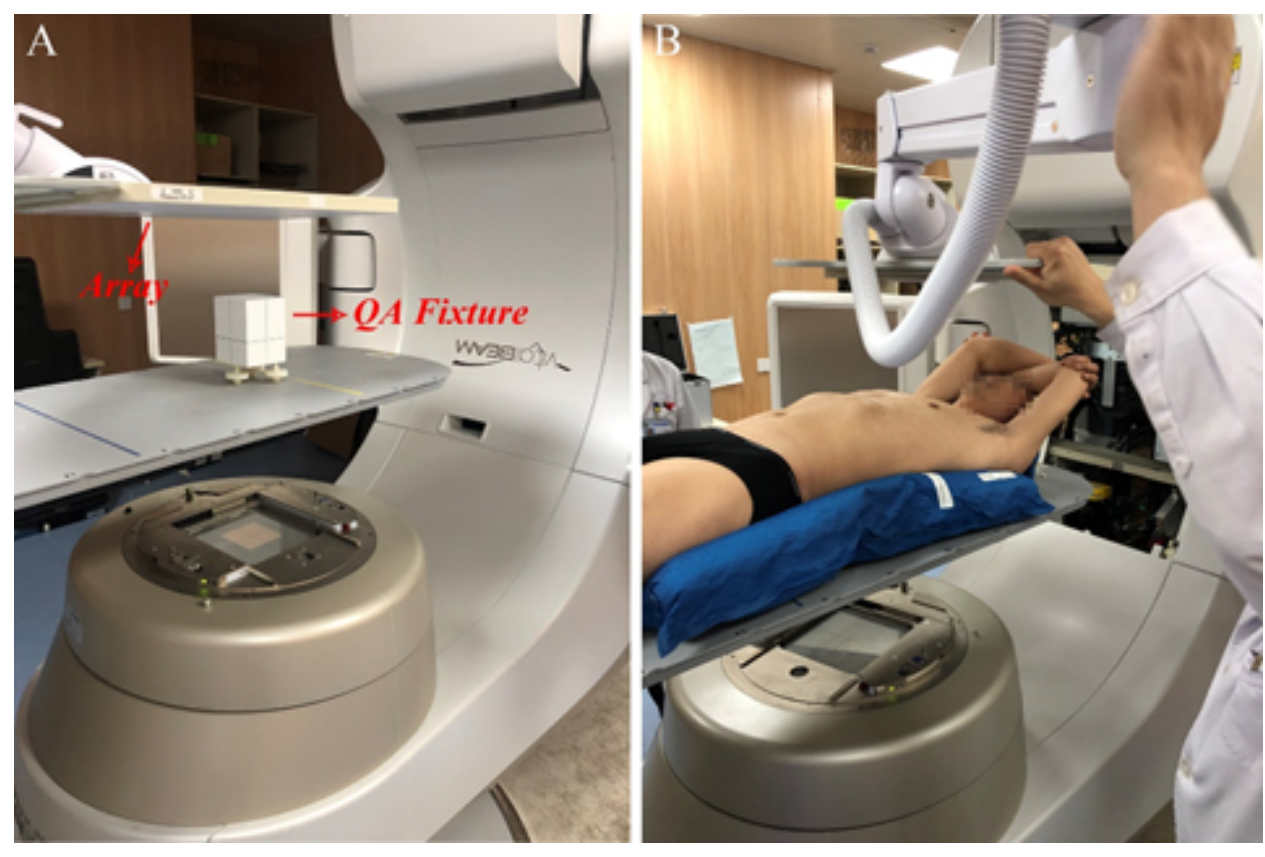

Figure 3

QA Fixture for A) QA procedure, B) patient and array setup 

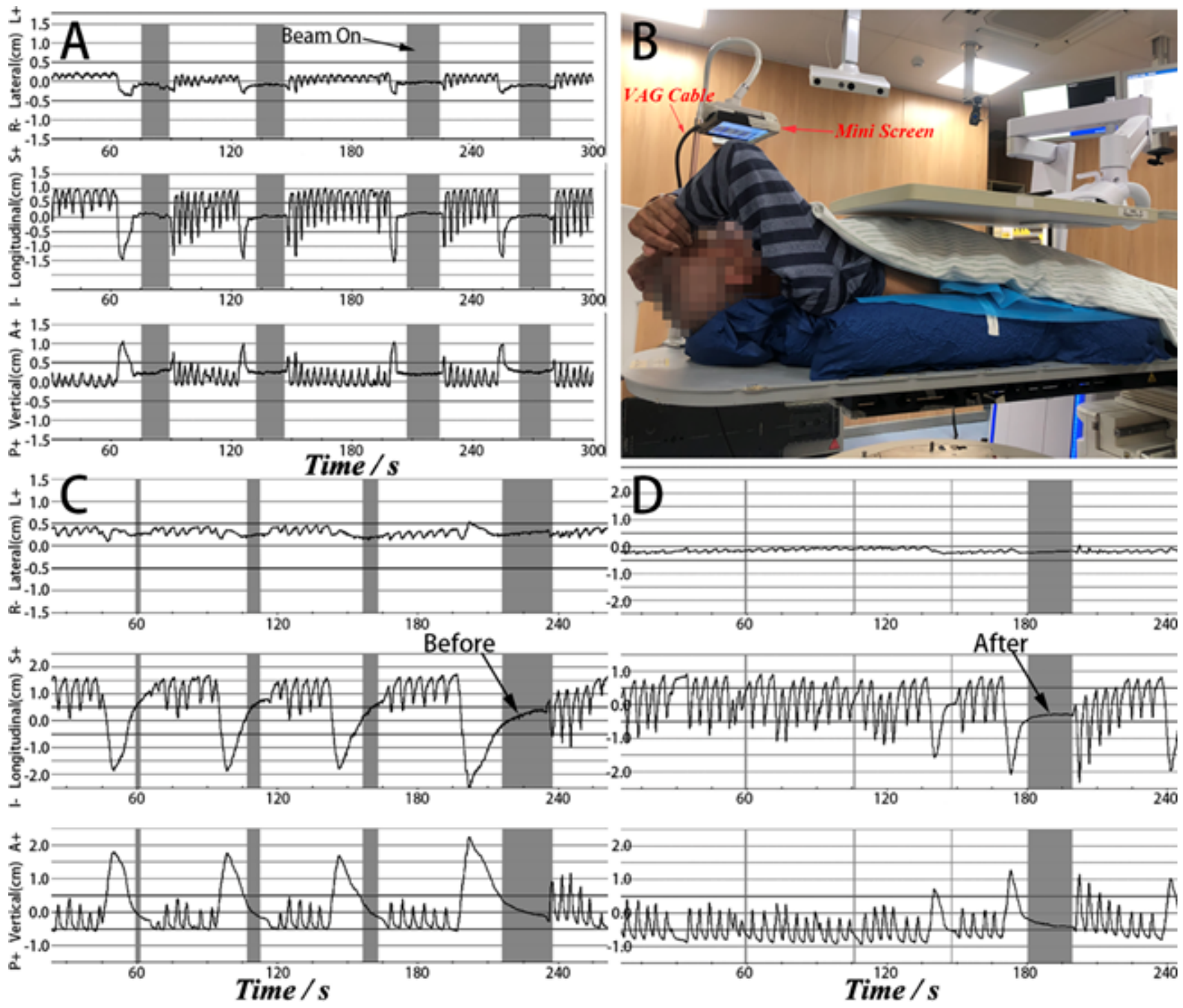

Figure 4

A) Recorded liver tumor motion at fraction 8 in the left-right (LR), infimum-supremum (IS), and anteriorposterior (AP) directions. Light gray areas indicate the time for the dose delivery. B) A Tracking Video Signal Synchronizing Device was fixed at the end of the couch and a mini screen were set above patient's head. C) and D) The recorded liver tumor motion before and after the setup of the tracking video signal synchronizing device. 